\title{
A 60 Second Periodic Modulation in Crab Pulsar Optical Light-Curve
}

\author{
Andrej Cadež and Mirjam Galičič \\ University of Ljubljana,FMF, Department of Physics, Slovenia
}

\begin{abstract}
The HST High Speed Photometer data and stroboscopic photometry data from the Asiago $1.82 \mathrm{~m}$ telescope (1994) show a $(0.0065 \pm$ 0.0015) magnitude weak modulation peak in Crab's light-curve at 60 seconds. Hubble data also suggest a phase modulation peak at the same period with the amplitude $(0.07 \pm 0.02)^{\circ}$. New stroboscopic data from December 1995 Asiago observations suggests the modulation amplitude $(0.0045 \pm 0.0015)$ magnitude, consistent with previous results.
\end{abstract}

\section{Introduction}

Our aim is to search for small-amplitude periodic modulation in Crab pulsar's light-curve on short time-scales. We observe the pulsar stroboscopically ${ }^{1}$ at the $1.82 \mathrm{~m}$ Asiago telescope. ${ }^{2}$ The Hubble data comprise of four approximately half an hour long HSP runs in visible light (Percival, 1993). Each of the data files gives the photon arrival time and the number of detected photons.

\section{Data analysis and results}

We selected two series of lowest noise observations from Asiago 1994 and 1995 and Fourier transformed the field stars' light-curves. The pulsar magnitude spectrum for 1994 displays a 3.3-sigma peak at the frequency $1 / 60 \mathrm{~s}\left(\nu_{\text {Asiago }}^{94}=\right.$ $(0.01673 \pm 0.00008) H z)$. The pulsar magnitude spectrum for 1995 has a 1.5 -sigma peak at the same frequency $\left(\nu_{\text {Asiago }}^{95}=(0.01668 \pm 0.00006) \mathrm{Hz}\right)$.

We Fourier analysed ${ }^{3}$ Hubble data for possible side-band modulation with frequency $\pm \Omega / 2 \pi$ above and below multiples of pulsar's rotational frequency. The average side-band spectrum of ten (we used 10 harmonics of pulsar's rotational frequency) displays a weak symmetrical peak at $\nu_{H u b b l e}=(0.01695 \pm$ $0.00029) \mathrm{Hz}$.

\footnotetext{
${ }^{1}$ The observing method and aparatus are described in (Čadež and Galičič, 1996a)

${ }^{2}$ Asiago and Padova Observatories, University of Padua, Italy

${ }^{3}$ Details of this analysis are described in (Čadež and Galičič, 1996b). Only data sets number 2 and 3 are included here since data sets number 1 and 4 exhibit much higher noise possibly due to higher HST jitter.
} 
The average modulation power spectrum is obtained by adding the normalized power spectra of all data sets and is shown in Figure 1. The peak at $1 / 60 \mathrm{~s}$ is 5-sigma high and no other peak is as prominent taking into account that at low frequencies the power spectrum has approximately 1 /frequency dependance which is a common result observed also in photometry of other field stars.

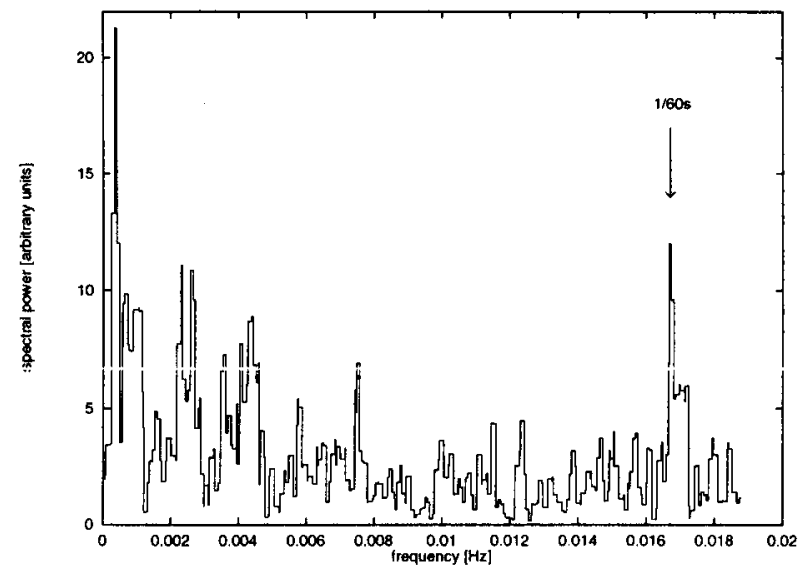

Figure 1. Average power spectrum: the sum of Asiago 94, Asiago 95 and HST Fourier spectra.

The existence of the 60-second period in Crab pulsar's optical light-curve is a tantalizing possibility. At least two explanations could be thought of. One of them-which drove our search-is the free precession of a young neutron star. A rough estimate (Lang, p278) suggests 120s for its period. The other frequency coming close into this range corresponds to the fundamental Alfven mode, and can be estimated (Lang, p307, p475) to be 1/20s.

Acknowledgments. This project is partially supported by EEC grant under contract PECO 94 n.ERBCIPDCT940028. We thank Asiago and Padua Astronomical Observatories and in particular Max Calvani for his encouragement. This work is based in part on observations with the HST obtained at the Space Telescope Science Institute, which is operated by AURA, Inc., under NASA contract NAS 5-26555. We thank HSP team, R. C. Bless, PI.

\section{References}

Cadež, A, Galičič, M. 1996a, A\&A, 306, 443

Čadež, A, Galičič, M. 1996b, Is the Crab Pulsar Showing a 60-second Periodic Modulation, presented at the conference Science with the Hubble Space Telescope, 1995, to be published in the conference proceedings

Lang, K. R. Astrophysical Formulae, Springer-Verlag, Berlin 1978

Percival, J. W. et al. 1993 ApJ, 407, 276 